\title{
Hidradenitis suppurativa in Crohn's disease during adalimumab therapy: a paradox?
}

\author{
Martina Emanuela ${ }^{1^{\star}}$, Campanati Anna $^{1^{\star}}$, Giuliodori Katia ${ }^{1 凶}$, Offidani Annamaria $^{1}$
}

\begin{abstract}
A sporadic association between hidradenitis suppurativa and other diseases is reported in the literature, but few authors have described the association with Crohn's disease. Adalimumab is a fully human monoclonal antibody targeted at tumor necrosis factor alpha approved for the treatment of Crohn's disease and, recently, for active moderate to severe hidradenitis suppurativa in adult patients that do not respond adequately to systemic conventional treatment. We report an unusual case of a paradoxical effect of adalimumab in the onset of hidradenitis suppurativa in a 40-year-old woman during the treatment of Crohn's disease.
\end{abstract}

Keywords: Crohn's disease, hidradenitis suppurativa, adalimumab, paradoxical effect

Received: 30 November 2016 | Returned for modification: 25 December 2016 | Accepted: 20 January 2017

\section{Introduction}

Crohn's disease (CD) is an inflammatory disorder of unknown etiology that primarily affects the gastrointestinal tract. Extraintestinal manifestations are observed in 20 to $40 \%$ of affected patients $(1,2)$. Hidradenitis suppurativa (HS) is an inflammatory and immunomodulating disorder of unknown etiology involving the pilosebaceous unit, the therapeutic approach to which is still a challenge for clinicians (3). It is characterized by relapsing painful abscesses, fistulas, and scarring. It occurs most frequently in apocrine gland-bearing areas such as the axilla, groin, perineal region, infra-mammary tissue, and scalp.

A sporadic association between HS and other diseases is reported in the literature, but few authors have described the association with $\mathrm{CD}$. The development of $\mathrm{CD}$ may precede the diagnosis of HS by an average of 3.5 years, suggesting a temporal relation. However the prevalence of HS and CD comorbidity is still debated (4).

Adalimumab is a fully human monoclonal antibody targeted at tumor necrosis factor alpha (TNF-alpha). It is approved for the treatment of moderate to severe psoriasis, psoriatic arthritis, rheumatoid arthritis, ankylosing spondylitis, juvenile idiopathic arthritis, and CD owing to its broad anti-inflammatory properties (5). Recently, on the basis of the results of several placebo-controlled studies (6), the European Medicines Agency (EMA) delivered a positive opinion on the use of adalimumab in the treatment of active moderate to severe HS in adult patients that do not respond adequately to systemic conventional treatment.

We report an unusual case of the onset of HS in a 40-year-old woman during adalimumab therapy for CD and enteropathic arthritis.

\section{Case report}

We report a case of 40-year-old non-smoking woman affected by $\mathrm{CD}$ and enteropathic spondylitis with a body mass index of 21 . She has been successfully treated since June 2013 with adalimumab 40 mg twice a week. Previously, she had been treated with methotrexate for only a few months with poor efficacy for articular pain with the onset of sideropenic anemia and increased liver parameters.
She had also been treated with infliximab infusion with a good response for joint and bowel disease, but this biological therapy was discontinued after the fourth infusion due to the paradoxical occurrence of diffuse plaque psoriasis. The cessation of infliximab therapy led to a complete resolution of her skin eruption.

In March 2015, during adalimumab therapy, the patient developed inflammatory deep nodules and cysts of the sinus tract and discharge of purulent fluid at the axillae and groin. She reported pain, swelling, and discomfort. HS, Hurley stage II, was clinically diagnosed for our patient (Figs. 1a, 1b).

We decided to treat her with minocycline $100 \mathrm{mg}$ once a day for 3 months; topically, she applied an antibiotic cream (clindamycin 1\% cream) and an antiseptic cleanser for 15 days, twice a day. The patient temporarily improved, but after a few days of discontinuing oral antibiotics the inflammatory skin lesions relapsed. In consultation with a rheumatologist and gastroenterologist, we decided to terminate the biological therapy.

The CD and related spondylitis were effectively treated with sulfasalazine $1 \mathrm{~g}$ twice a day.

In June 2015, after 3 months of oral antibiotics, the active inflammatory abscesses were entirely healed and had evolved into scars; there was neither purulent secretion nor pain (Figs. 2a, 2b).

\section{Discussion}

The most common extra-intestinal manifestation in inflammatory bowel disease (IBD) patients is joint involvement, with a prevalence between 17 and 39\%. It is frequently characterized by an involvement of the axial joints, but may also be associated with peripheral arthritis (synovitis and/or dactylitis and/or enthesopathy) (7).

TNF-alpha inhibitors should be considered the first-line therapeutic approach when moderate-to-severe luminal CD or ulcerative colitis (UC) is associated with polyarthritis (8).

Dermatologic comorbidities in patients with IBD are not rare and their early recognition is very important for treatment, also because they may rapidly worsen. These comorbidities include pyoderma gangrenosum, erythema nodosum, HS, and psoriasis. Zippi $\mathrm{M}$ et al. recently focused on the efficacy of biological therapies in managing these skin conditions (9). 


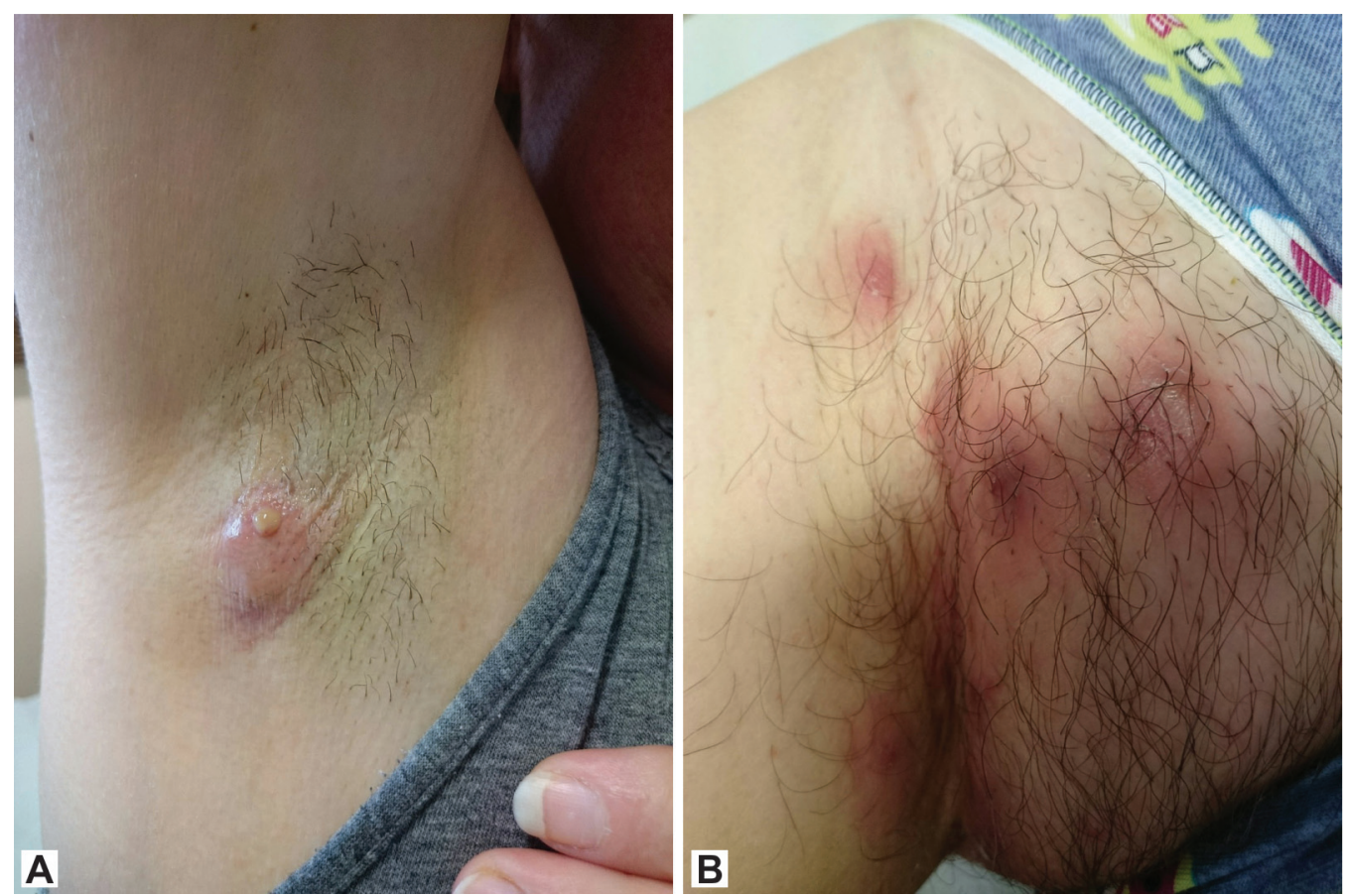

Figure $1 \mathrm{a}, \mathrm{b} \mid$ Nodules and cysts in the sinus tract and discharge of purulent fluid at the axillae and groin.
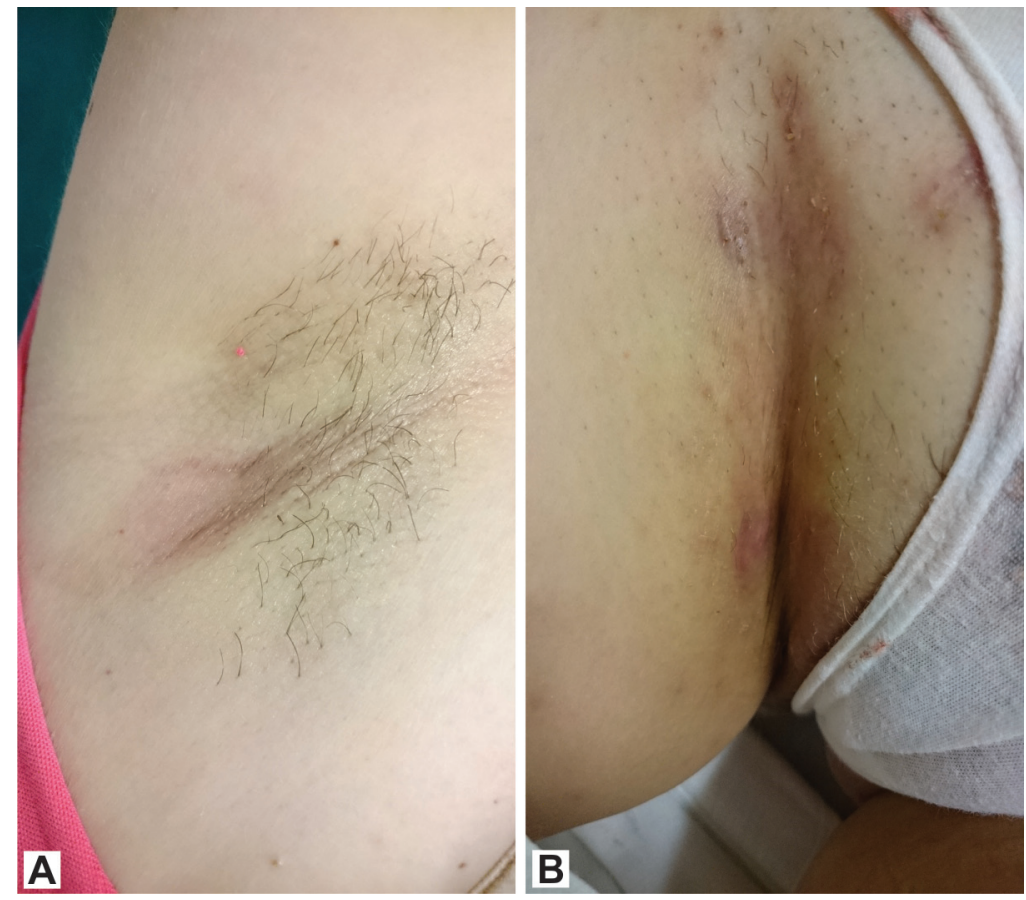

Figure $2 \mathrm{a}, \mathrm{b}$ | After therapy there is no evidence of purulent secretion and a marked reduction of erythema.

Indeed, the association between IBD and HS is still under discussion. In 2010, Van der Zee et al. submitted a questionnaire to 1,093 patients with IBD. Altogether, 688 had CD (63\%) and 405 (37\%) had UC. Overall, 255 patients with IBD reported having HS (23\%), with $26 \%$ of those with CD and $18 \%$ of those with UC having HS (3). However, they admitted that the data were inadequate for establishing a comorbidity closely linked with IBD. More recently, Janse et al. evaluated the prevalence of HS in 1,260 patients with IBD: $6.8 \%$ to $10.6 \%$ versus $1 \%$ to $2 \%$ in the general population. Female sex, smoking, a higher body mass index, and younger age were independent associated parameters for HS. They also observed two new associations in genomic regions that need further investigations to be confirmed (10).

In recent years, confirmation of a connection between HS and IBD was also suggested by Diamantova et al., who proposed a common therapy with adalimumab to treat the co-occurrence of
CD and HS in a patient also suffering from pyoderma gangrenosum (11). These data are also confirmed by Kalman, who showed the efficacy of infliximab and adalimumab in a large number of cases presenting HS and CD (12). Thus, the literature suggests a common pathogenetic pathway that is a target for the same biological drugs.

In our case, the therapeutic management of the patient was a greater challenge than usual because HS had developed during the administration of a TNF alpha inhibitor.

The patient had no risk factors associated with developing HS (no family history, no overweight or obesity, and no cigarette smoking). Thus, the appearance of HS in a patient affected by CD during anti TNF-alpha therapy can be detected as a comorbidity of IBD with a loss of efficacy of the biological agent, but also as a paradoxical effect of this therapy.

Paradoxical effects of anti TNF-alpha drugs represent a new 
onset or exacerbation of a condition that is normally improved through the use of the same biological therapy; psoriasis, psoriasiform eruption, and eczema are the most frequently observed. The causal mechanism is still a matter of debate, but may implicate an imbalance of cytokines toward interferons, chemokines, and probably IL-17 (13). In particular, the inhibition of TNF alpha by antibody molecules may stimulate increased maturation of dermal plasmacytoid dendritic cells from hematopoietic progenitors with uncontrolled production of interferon-gamma (IFN-gamma). Histological stains have also shown an overproduction of IFNgamma expressing Th1 lymphocytes and IL17-IL22- expressing Th17 lymphocytes. (14).

Recently, Faivre et al. (15) published a multicenter nationwide retrospective study describing the clinical characteristics and outcome of patients that were affected by a chronic inflammatory disease and developed paradoxical HS under biological agents (BA). The enrollment was achieved by asking physicians (rheumatologists and dermatologists) to report all cases of HS and complete an on-line standardized questionnaire. The authors collected 25 cases of HS onset after biological agent administration; interestingly, half of the patients that received TNF-alfa inhibitors $(\mathrm{n}=$ 11 of 22) showed another autoimmune disease (psoriasis or CD, alopecia areata) concurrently or within 1 year of HS onset. Adalimumab was administered in $48 \%$ of cases of new HS onset, but this TNF-alfa inhibitor is the most frequently prescribed in inflammatory diseases. The authors propose an interesting management strategy, suggesting continuation of BA therapy in mild HS, obviously with the addition of specific HS treatments.

Reading the literature, there is actually no consensus as to whether to continue or discontinue anti-TNF-a therapy in these cases (16). In the case series by Delobeau et al., three of the four patients were able to continue adalimumab therapy, whereas one patient required a switch to ustekinumab (17). Two cases of paradoxical HS during adalimumab therapy for CD, described by Harvin and Kasarala, showed how difficult the therapeutic management of these patients is because they often have a gastrointestinal disease well controlled by BA (18). We suggest deciding on a case-by-case basis, considering the effectiveness of biological therapy, alternative therapeutic choices, and the severity of side effects if they are recalcitrant to traditional treatment.

This case interestingly involves a probable new "paradoxical" effect of adalimumab during the treatment of CD and suggests the need to learn more about the etiology and pathogenetic mechanism of both diseases; it also demonstrates the broad spectrum of side effects of anti TNF-alpha agents and the challenge of treating them. Finally, it would be interesting to outline the profile of a "paradoxical patient" in order to choose the best treatment with minimal side effects.

The fact that our patient had already had a previous paradoxical manifestation after treatment with other anti-TNF-alpha molecules strengthens the hypothesis that HS is a paradoxical effect of adalimumab therapy, indicating a possible paradoxical genetic profile for this patient. Thus, in this case, the onset of psoriasis and HS may be considered a class effect of anti-TNF-alpha agents; that is, a paradoxical response of our patient to drugs with a similar chemical structure, mechanism of action, and pharmacological effects, rather than a drug-specific adverse event.

\section{References}

1. Trikudanathan G, Venkatesh PG, Navaneethan U. Diagnosis and therapeutic management of extraintestinal manifestations of inflammatory bowel disease. Drugs. 2012;72:2333-49.

2. Ganzetti G, Campanati A, Offidani A. Alopecia areata: a possible extraintestinal manifestation of Crohn's disease. J Crohns Colitis. 2012;6:962-3.

3. Martina E, Offidani A. Hidradenitis suppurativa: how to treat with BoNT-A. New York: Nova Science Publishers, Inc.; 2015.

4. Van der Zee HH, van der Woude CJ, Florencia EF, Prens EP. Hidradenitis suppurativa and inflammatory bowel disease: are they associated? Results of a pilot study. Br J Dermatol. 2010;162:195-7.

5. Prattichizzo F, Giuliani A, Recchioni R, Bonafè M, Marcheselli F, De Carolis S, et al. Anti-TNF-a treatment modulates SASP and SASP-related microRNAs in endothelial cells and in circulating angiogenic cells. Oncotarget. 2016;15;7:11945-58.

6. Kimball AB, Kerdel F, Adams D, Mrowietz U, Gelfand JM, Gniadecki R, et al. Adalimumab for the treatment of moderate to severe hidradenitis suppurativa. Ann Intern Med. 2012;157:846-55.

7. Peluso R, Di Minno MN, lervolino S, Manguso F, Tramontano G, Ambrosino P, et al. Enteropathic spondyloarthritis: from diagnosis to treatment. Clin Dev Immunol. 2013;2013:631408.

8. Peluso R, Manguso F, Vitiello M, lervolino S, Di Minno MN. Management of arthropathy in inflammatory bowel diseases. Ther Adv Chronic Dis. 2015; 6:65-77.

9. Zippi M, Pica R, De Nitto D, Paoluzi P. Biological therapy for dermatological manifestations of inflammatory bowel disease. World J Clin Cases. 2013; 16;1:74-8.

10. Janse IC, Koldijk MJ, Spekhorst LM, Vila AV, Weersma RK, Dijkstra G, et al. Identification of clinical and genetic parameters Associated with hidradenitis suppurativa in inflammatory bowel disease. Inflamm Bowel Dis. 2016;22:106-13.

11. Diamantova D, Lomickova I, Cetkovska P. Adalimumab treatment for hidradenitis suppurativa associated with Crohn's disease. Acta Dermatovenerol Croat. 2014;22:291-3.

12. Kamal N, Cohen BL, Buche S, Delaporte E, Colombel JF. Features of patients with Crohn's Disease and hidradenitis suppurativa. Clin Gastroenterol Hepatol. 2016;14:71-9.

13. Wendling D, Prati C. Paradoxical effects of anti-TNF-a agents in inflammatory diseases. Expert Rev Clin Immunol. 2014;10:159-69.

14. Tillack C, Ehmann LM, Friedrich M, Laubender RP, Papay P, Vogelsang H, et al. Anti-TNF antibody-induced psoriasiform skin lesions in patients with inflammatory bowel disease are characterised by interferon-y-expressing Th1 cells and IL17A/IL-22-expressing Th17 cells and respond to anti-IL-12/IL-23 antibody treatment. Gut. 2014; 63:567-77.

15. Faivre C, Villani AP, Aubin F, Lipsker D, Bottaro M, Cohen JD, et al. Hidradenitis suppurativa (HS): an unrecognized paradoxical effect of biologic agents (BA) used in chronic inflammatory diseases. J Am Acad Dermatol. 2016;74:1153-9.

16. Denadai R, Teixeira FV, Saad-Hossne R. The onset of psoriasis during the treatment of inflammatory bowel diseases with infliximab: should biological therapy be suspended? Arq Gastroenterol. 2012;49:172-6.

17. Delobeau M, Abdou A, Puzenat E, Deveza E, Biver-Dalle C, van de Laak A, et al. Observational case series on adalimumab-induced paradoxical hidradenitis suppurativa. J Dermatolog Treat. 2016;27:251-3.

18. Harvin G, Kasarala G. Two cases of paradoxical hidradenitis suppurativa while on adalimumab. Case Rep Gastroenterol. 2016;10:88-94. 